\title{
A rare case; isolated tubal torsion
}

\author{
Onur Karaaslan ${ }^{1 *}$, Dogukan Yildirim ${ }^{2}$, Erkan Şimşek ${ }^{3}$, Latif Hacıoğlu ${ }^{1}$ \\ ${ }^{1}$ Department of Obstetrics and Gynecology Y uzuncu Yal University, Van, Turkey \\ ${ }^{2}$ Department of Obstetrics and Gynecology Elazig Public Hospital, Elarig, Turkey \\ ${ }^{3}$ Department of Obstetrics and Gynecology Tunceli Public Hospital, Tunceli, Turkey
}

\begin{abstract}
Although isolated torsion of the tuba is very rare, it should be kept in mind especially in women of reproductive age who present with abdominal pain, as it causes fertility problems especially in late diagnosed cases. Pre-operative diagnosis is difficult because there is no pathognomonic symptom or specific clinical, imaging and laboratory findings. Therefore, it should be considered in reproductive age women presenting with undiagnosed abdominal pain.
\end{abstract}

Key Words: Tubal torsion, fertility, undiagnosed abdominal pain

\section{Introduction}

Tubal torsion, which is a very rare cause of gynecological emergency, is observed at a rate of approximately $1 / 1.500 .000(1,2)$. It was first described by Bland-Sutton in 1890 (3). The majority of the cases were women in the reproductive period. No diagnostic symptoms, clinical signs, laboratory markers, or pathognomonic radiological features were identified in these cases (4). Its etiology is examined in two groups as intrinsic and extrinsic causes (5). Intrinsic causes include congenital anomalies of the tuba, hydrosalpinx, hematosalpinx, tubal neoplasms, tubal ligation or tubal specific factors such as tubal surgical interventions; whereas ovarian and paratubal masses, pregnancy, trauma, adhesions, pelvic congestion and sudden body movements are extrinsic causes. Although tubal torsion is rare, early diagnosis is important. Late diagnosis can lead to necrosis and irreversible damage. Although the function of the detorsion tuba is debatable, preventive surgery is recommended unless the tuba is completely necrotic (6). In this case report, we discuss a case of isolated tubal torsion operated at our clinic.

\section{Case Report}

A 32-year-old patient with 2 normal vaginal deliveries and an abortion was admitted to an external health care facility and followed up for 2 days. She was then referred our hospital because of sudden onset abdominal pain along with nausea and vomiting. She stated that the pain started suddenly, became stronger and nausea and vomiting had been added for the last 6 hours. In the examination of the patient, there was defense and rebound in the right lower quadrant. Her vital signs were normal. Laboratory tests revealed wbc 16,000 , hb 12.5 , hct 36.1. Other laboratory parameters were within normal ranges. An anechoic cystic mass of approximately $6 \mathrm{~cm}$ was observed in the right adnexal area in the transvaginal USG. Bilateral ovaries were clear and there was minimal free fluid in the abdomen. Doppler USG showed blood flow around the cystic mass, but no blood flow through the mass. The abdominal USG was performed with a prediagnosis of acute abdomen, the result of the abdominal usg was reported as ananechoic cystic mass of approximately $5.5 \mathrm{~cm}$ in size adjacent to the right ovary. Diagnostic and operative laparoscopy was planned with the prediagnosis of acute abdomen and adnexal torsion. During the operation, uterine left tuba and ovary were normal. It was observed that the right tuba was twisted by turning 2 times around itself and had a necrotic mass approximately $6-7 \mathrm{~cm}$ in size (Figure 1,2). Firstly, the tubal torsion was detorsion and the arterial flow was expected to return again. Hemorrhagic necrosis was reported as a severe acute inflammatory reaction consistent with tubal torsion. The patient was discharged on the first postoperative day without any postoperative complications.

\section{Discussion}

Although isolated tubal torsion is very rare, it can be seen in premenarchal, postmenopausal and gestational periods as well as in women of reproductive age, as in our case $(7,8,9)$. Isolated tubal torsion has no specific clinical, laboratory and imaging findings (10). It is most commonly presented with lower abdominal pain, which may be blunt, continuous, paroxysmal 


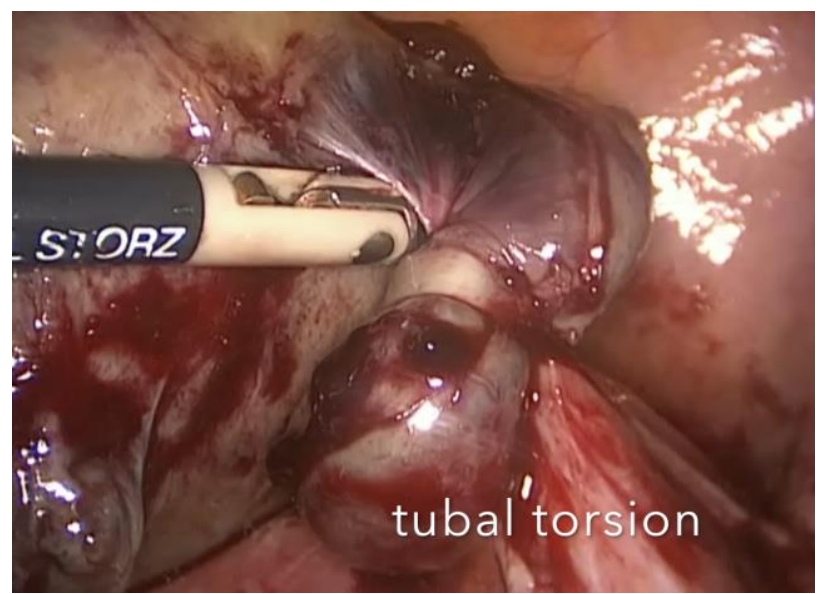

Fig. 1. Spontaneous tubal torsion twice

and may spread to the leg or hip (11). Current findings may be accompanied by nausea, vomiting and peritoneal irritation findings (12). Although USG is not very helpful in differential diagnosis, it is reported that the presence of diastolic reverse flow and high impedance waveforms in the affected tuba is diagnostic in color Doppler USG examination (13). These specific doppler findings may not be seen in cases of delayed and possibly absence of blood circulatory. Normal doppler USG findings do not rule out the definition of torsion (14). While the diagnosis can be made only in $20 \%$ of preoperative cases, the definitive diagnosis usually requires laparoscopy or laparotomy (7). In most of the cases published in the literature, torsion was seen in the right tuba, and it was suggested that the left tuba was close to the sigmoid colon and was less mobile (15). Because of the pain pattern and other symptoms, ovarian torsion, ovarian cyst rupture, appendicitis, ectopic pregnancy rupture, pelvic inflammatory disease, intestinal surgical pathologies, urolithiasis and acute cystitis are considered in the differential diagnosis (16). Pelvicinflammatory disease was not considered with the present findings and symptoms. Isolated tubal torsion was observed in the right tuba. The blood supply of the ovary is not expected due to both uterine and ovarian arteries. In our case, both ovaries were found to be normal (9). In torsion cases, when the torsion is fixed before the onset of gangrenous changes, the tubas can be preserved. Therefore, early diagnosis is important. In the study of Mazouni et al., it was emphasized that more than 10 hours between the onset of pain and surgery increased the risk of tubal necrosis (17). In some sources, detorsion is recommended, even if the appearance is necrotic. If necrotic tissue is present in some sources, it is recommended to remove the existing necrotic tissue as detorsion of the tuba may lead to thrombosis $(6,18)$. There is not enough information about the effect of tubal function and fertility after detorsion

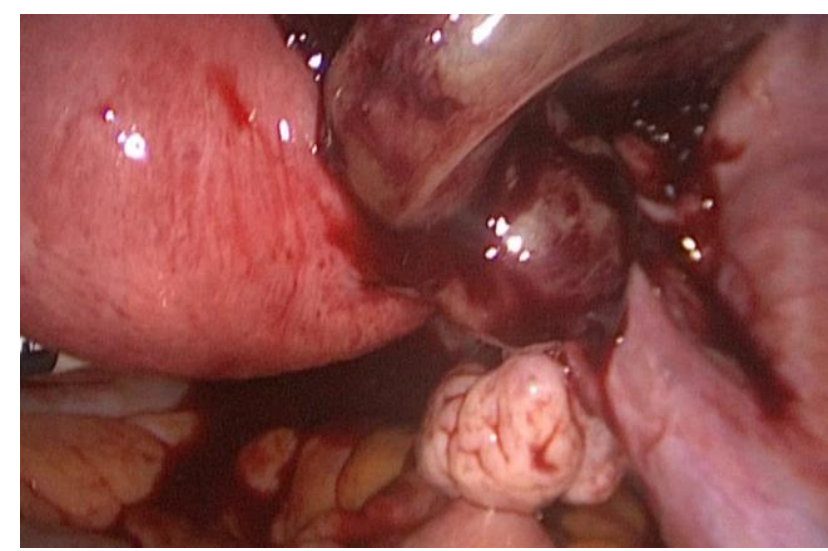

Fig. 2. Spontaneous tubal torsion, chronic inflammation

cases. With the available information, salpingectomy is the recommended treatment in cases with delayed gangrenous changes, whereas detorsion is recommended in cases without gangrenous changes. Therefore, early diagnosis and treatment is important for the protection of tuba especially in reproductiveage women. Although it is a very rare condition, isolated tubal torsion should be considered in the differential diagnosis in acute onset abdominal pain.

\section{References}

1. Antoniou N, Varras M, Akrivis C, Kitsiou E, Stefanaki S, Salamalekis E. Isolated torsion of the fallopian tube: a case report and review of the literature. ClinE xp obstet Gynecol 2004; 31: 235238.

2. Ho PL, LiangSJi Su HW, Chang CY, Hsu CS, Ling TH. Isolated torsion of the fallopian tube: a rare diagnosis in an adolescent without sexual experience. Taiwan J Obstet Gynecol 2008; 47: 235-237.

3. Sutton JB. Salpingitis and some of its effects. Lancet 1890; 2: 1146-1148.

4. Krissi H, Shalev J, Bar-Hava I. Fallopian tube torsion: laparoscopic evaluation and treatment of a rare gynecological entity. J Am Board Fam Pract 2001; 14: 274-277.

5. Youssef AF, Fayad MM, Shafeek MA. Torsion of the fallopian tube. A clinico- pathological study. Acta Obstet Gynecal Scand 1962; 41: 292-309.

6. Raziel A, Mordecahai E, Friedler S, Schachter M, Pansky M, Ron-el R. Isolated recurrent torsion of the fallopian tube. Hum Reprod 1999; 14: 30003001.

7. Comerci G, Colombo FM, Stefanetti M, Grazia G. Isolated fallopian tube torsion: a rare but important event for women of reproductive age. Fertil Steril 2008; 90: 1198: 23-25.

8. Lima M, Libri M, Aquino A, Gobbi D. Bilateral hydrosalpinx with asynchronous tubal torsion: an exceptional finding in a premearchial girl. J Pediatr Surg 2011; 46: 27-29. 
9. Phupong V, Intharasakda P. Twisted fallopian tube in pregnancy: a case report. BMC Pregnancy Childbirth 2001; 1: 5.

10. Chang HC, Bhatt S, Dogra VS. Pearls and pitfalls in diagnosis of ovarian torsion. Radiographics 2008; 28: 1355-1368.

11. Lo LM, Chang SD, Lee CL, Liang CC. Clinical manifestations in women with isolated fallopian tubal torsion; a rare but important entity. Aust N Z J ObstetGynecol 2011; 51: 244-247.

12. Bharathi A, Gowri M Torsion of the fallopian tube and the hematosalpinx in perimenopausal womena case report. J ClinDiagnRes 2013; 7: 731-733.

13. Lineberry TD, Rodriguez $H$. Isolated torsion of the fallopian tube in an adolescent: A case report. J Pediatr AdolescGynecol 2000; 13: 135-138.
14. Rha SE, Byun JY, Jung JI, Choi BG, Kim BS, Kim H, Lee JM. CT and MR imaging features of adnexal torsion. Radiographics 2002; 22: 283-294.

15. Milki A, Jacobson DH. Isolated torsion of the fallopian tube. A case report. J ReprodMed 1998; 43: 836-838.

16. Gross M, Blumstein SL, Chow LC. Isolated fallopian tube torsion: A rare twist on a common theme. Am J Radiology 2005; 185: 1590-1592.

17. Mazouni C, Bretelle F, Menard JP, Blanc B, Gamerre M Diagnosis of adnexal torsion and predictive factors of adnexal necrosis. Gynecol Obstet Fertil 2005; 33: 102-106.

18. Breitowicz B, Wiebe BM, Rudnicki M. Torsion of bilateral paramesonephric cysts in young girls. Acta Obstet Gynecol Scand. 2005; 84: 199-200. 